\title{
RNA secondary structures in Dscam1 mutually exclusive splicing: unique evolutionary signature from the midge
}

\author{
WEILING HONG, YANG SHI, BINGBING XU, and YONGFENG JIN \\ MOE Laboratory of Biosystems Homeostasis and Protection and Innovation Center for Cell Signaling Network, College of Life Sciences, \\ Zhejiang University, Hangzhou, Zhejiang, ZJ310058, China
}

\begin{abstract}
The Drosophila melanogaster gene Dscam 1 potentially generates 38,016 distinct isoforms via mutually exclusive splicing, which are required for both nervous and immune functions. However, the mechanism underlying splicing regulation remains obscure. Here we show apparent evolutionary signatures characteristic of competing RNA secondary structures in exon clusters 6 and 9 of Dscam 1 in the two midge species (Belgica antarctica and Clunio marinus). Surprisingly, midge Dscam 1 encodes only $\mathbf{6 0 0 0}$ different isoforms through mutually exclusive splicing. Strikingly, the docking site of the exon 6 cluster is conserved in almost all insects and crustaceans but is specific in the midge; however, the docking site-selector base-pairings are conserved. Moreover, the docking site is complementary to all predicted selector sequences downstream from every variable exon 9 of the midge Dscam1, which is in accordance with the broad spectrum of their isoform expression. This suggests that these cis-elements mainly function through the formation of long-range base-pairings. This study provides a vital insight into the evolution and mechanism of Dscam1 alternative splicing.
\end{abstract}

Keywords: alternative splicing; Dscam; base-pairing; evolution; midge

\section{INTRODUCTION}

Pre-mRNA alternative splicing produces multiple proteins by a single gene, which greatly increases protein diversity (Nilsen and Graveley 2010; Matera and Wang 2014; Lee and Rio 2015). Alternative splicing could mediate cell differentiation and growth, and splicing dysregulation has the ability to alter the proteomic diversity of many diseases (Scotti and Swanson 2016; Wang et al. 2020). The most striking case is the Drosophila melanogaster Down syndrome cell adhesion molecule (Dscam1) gene, which potentially generates up to 38,016 isoforms through the mutually exclusive splicing of four exon clusters 4, 6, 9, and 17 (Schmucker et al. 2000). Many studies have demonstrated that Dscam1 isoforms are required for nervous and immune function (Watson et al. 2005; Zipursky and Sanes 2010; He et al. 2014).

Studies indicate that the presence of competing RNA secondary structures, between the docking site and selector sequences, are thought to guarantee that only one variant exon is selected from a cluster of exons in insect Dscam 1 (Graveley 2005; Anastassiou et al. 2006; Yang et al. 2011; Xu et al. 2019). In the exon 6 cluster, the docking site is found in the intron downstream from exon 5 and

Corresponding author: jinyf@zju.edu.cn

Article is online at http://www.rnajournal.org/cgi/doi/10.1261/rna. 075259.120 can pair with the selector sequences upstream of each exon 6 variant (Graveley 2005). Except for RNA secondary structures, RNA binding proteins (i.e., the heterogeneous nuclear ribonucleoprotein hrp36) and cis-elements (i.e., locus control region, LCR) were shown to be involved in exon 6 inclusion (Olson et al. 2007; Wang et al. 2012). Similar docking site-selector base-pairings have been identified in the exon 4 and exon 9 clusters of Dscam 1 and in other invertebrate and vertebrate genes (Yang et al. 2011; Suyama 2013; Yue et al. 2016b, 2017; Hatje et al. 2017; Pan et al. 2018). However, docking site-selector base-pairings in the exon 4 and 9 clusters are not readily apparent or as conserved as the exon 6 cluster of fly Dscam 1. Very recently, some researchers argued against a long-range base-pairing mechanism for exon 4 and 9 selection because of a lack of conserved intron sequences or basepairing in the variable exon 4 and 9 clusters (Haussmann et al. 2019; Ustaoglu et al. 2019). Due to technical challenges associated with the large size and complexity of the exon cluster, an alternative solution to this dilemma is to identify more examples with highly apparent evolutionary signatures of docking site-selector base-pairings.

(c) 2020 Hong et al. This article is distributed exclusively by the RNA Society for the first 12 months after the full-issue publication date (see http://rnajournal.cshlp.org/site/misc/terms.xhtml). After 12 months, it is available under a Creative Commons License (Attribution-NonCommercial 4.0 International), as described at http://creativecommons.org/ licenses/by-nc/4.0/. 
The rapidly increasing availability of genomic information regarding insects, particularly species such as the Antarctic midge (Belgica Antarctica) (Kelley et al. 2014), which is the only insect endemic to Antarctica, will help to enhance our understanding of the evolution and mechanism of Dscam 1 alternative splicing.

In this study, we reveal apparent evolutionary signatures of competing RNA secondary structures in the exon clusters 6 and 9 of Dscam 1 in two species of Antarctic midges (Belgica antarctica and Clunio marinus). Compared to other fly species, these midge species encode only around one-sixth of Dscam1 isoforms, representing the smallest Dscam 1 splice repertoire known in insects. Strikingly, the docking site of the exon 6 cluster is conserved in almost all insects and crustaceans but is unique in the midge; however, the docking site-selector base-pairings are conserved. Moreover, we found that the docking site is complementary to all predicted selector sequences downstream from every variable of exon 9 in the midge Dscam 1 gene, which is in accordance with the broad spectrum of isoform selection. This study supports the notion that com- peting RNA secondary structures are a crucial player in regulating Dscam1 alternative splicing.

\section{RESULTS AND DISCUSSION}

\section{The midge Dscam1 gene encodes only $\sim 3000$ ectodomain isoforms}

To decipher the mechanism and function of Dscam1 isoform diversity, we characterized the Dscam1 gene in two midge species (B. antarctica and C. marinus). Comparative analysis showed that midges shared similar overall organization of Dscam 1 with other dipteran species, albeit with a striking difference in the number of variable exons (Fig. 1A). The B. antarctica Dscam1 gene contained 13, 12,21 , and 2 exon variants in the exon clusters 4, 6, 9, and 17, respectively. Therefore, only 6552 distinct isoforms might be theoretically generated through mutually exclusive splicing in four variable exon clusters. The Clunio marinus Dscam 1 gene could potentially encode 3000 $(10 \times 15 \times 20)$ different ectodomains linked to two

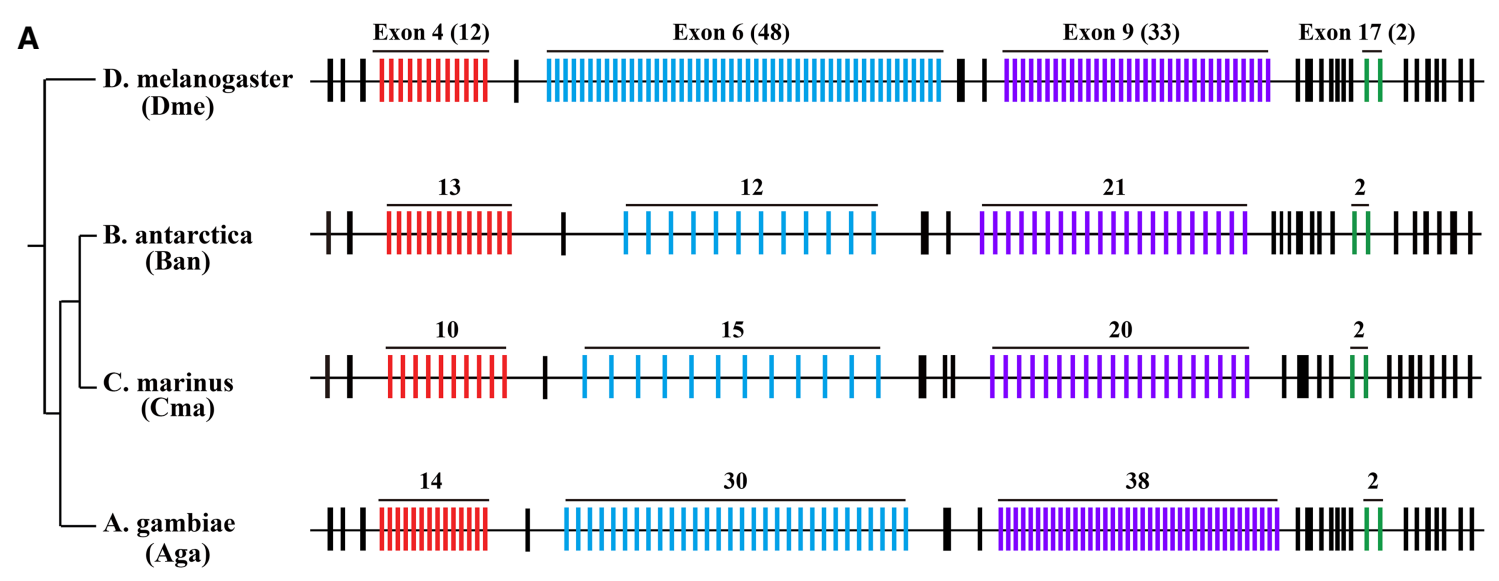

B

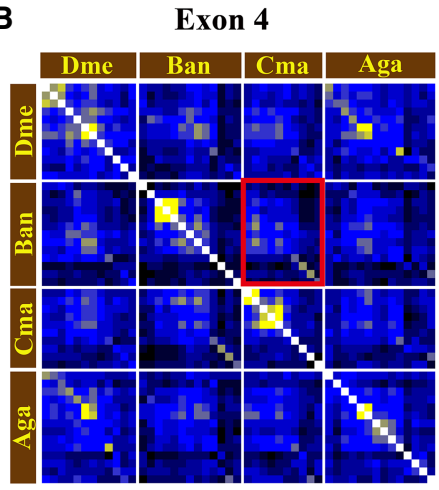

Exon 6

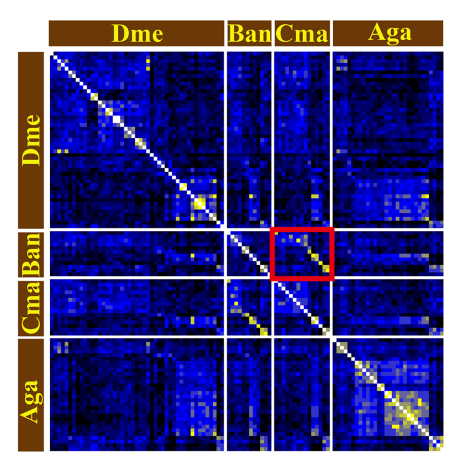

Exon 9

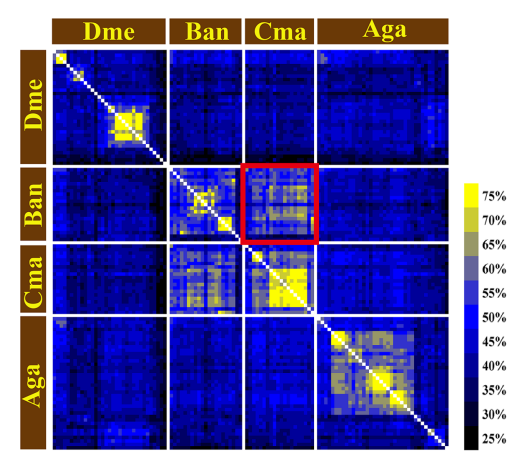

FIGURE 1. The organization of midge Dscam1 genes and variable exon similarity. (A) Schematic diagram of the Dscam1 gene structures in the two midges (B. antarctica and C. marinus). Midges encode much less Dscam1 ectodomain isoforms than D. melanogaster and A. gambiae. (B) Heatmaps of exon sequence similarity within the exons 4 (left), 6 (middle), and 9 (right) clusters in the midge Dscam1. D. melanogaster and $A$. gambiae were selected as representative species of the fly and Culicidae clades, respectively. To simplify visualization, the variable exons are depicted in the same linear order in which they reside in each cluster. Yellow indicates a high percent identity, while black indicates a low or little sequence similarity. The exon sequence similarity between $B$. antarctica and $C$. marinus is highlighted by red box. White boxes indicate pairs with $100 \%$ similarity between the same exons. 
alternative transmembrane domains. The data in these two midge species represent the smallest Dscam 1 splice repertoires known in insects. Compared with other dipterans, the number of midge variable exons changes in a clusterspecific manner. The number of variable exon 4 changes in midges was comparable to that of other dipteran species. In contrast, the number of variable exon 9 changes in midge was reduced by $0.5-1$-fold. Most strikingly, the Antarctic midge contained only 12 variable exon $6 \mathrm{~s}$, only a quarter of that of $D$. melanogaster. For most species, the exon 6 cluster contains the largest number of variable exons among the exon 4, 6, and 9 clusters. However, in the Antarctic midge Dscam1 gene, the exon 6 cluster had fewer variable exons than the exon 4 and 9 clusters (Fig. 1A). This suggests that the midge exon 6 cluster might be subject to specific evolutionary constraints during speciation or these might be driven by adaptation to an extreme environment.

\section{Rapid evolution of the variable exons in the midge Dscam1 gene}

Given the striking reduction of midge Dscam 1 isoforms mentioned above, we were interested in determining the extent to which the variable exons have evolved. To address this, we globally analyzed the variable exon relationships across three major clades of the midge, fly and Culicidae. We selected D. melanogaster and Anopheles gambiae to represent the fly and Culicidae clades, respectively. For exon clusters 4, 6, and 9, the pairwise identity of each variable exon to all other variable exons was analyzed. Interestingly, we observed only two 1:1 orthologous exon 4 pairs among the fly, Culicidae, and midges (left panel, Fig. 1B; Supplemental Fig. S1). Moreover, only half of the variable exon $4 \mathrm{~s}$ in $C$. marinus were orthologous to exons in B. Antarctica, as revealed by the yellow diagonals in approximately the same linear order in the heatmap panel comparing the two species (highlighted in the left panel, Fig. 1B; Supplemental Fig. S1A). This was in contrast to the previous observation that most exon $4 \mathrm{~s}$ within a species are orthologous to exons in the fly and Culicidae species (Fig. 1B; Lee et al. 2010). This suggests that the exon 4 cluster might have diverged rapidly during midge evolution.

A similar trend was observed in the study of the midge exon 9 clusters. Little evidence was found to indicate the presence of orthologous exon 9 pairs between B. Antarctica and C. marinus (right panel, Fig. 1B). Instead, high similarities exist between the exon 9s in different species (e.g., exons 9.9-9.18 in B. antarctica and exons 9.8-9.18 in C. marinus), which involve several large blocks of strongly similar variable exons within each midge species, rather than pairs of variable exons (highlighted in the right panel, Fig. 1B; Supplemental Fig. S2). This suggests that an ancestral exon 9 was independently expand- ed via multiple rounds of duplications in these two midge species. However, analysis of the variable exons of the exon 6 cluster showed a different pattern. In contrast with the exon clusters 4 and 9, most variants within the exon 6 cluster showed a clear pairing between $B$. antarctica and C. marinus, as revealed by the yellow diagonals in approximately the same linear order (highlighted in the middle panel, Fig. 1B; Supplemental Fig. S3). This suggests that these midge exons emerged before the divergence of the $B$. antarctica and $C$. marinus. However, only a few 1:1 orthologous pairs in exon 6 were observed between the fly and the midge. Overall, these data indicate that the variable exons of midge Dscam 1 evolved rapidly during speciation.

\section{The docking site of the exon 6 cluster is midge- specific, but RNA secondary structures are conserved}

Next, we sought to determine whether the arrangement of the docking site and selector sequences and their secondary structures in Drosophila are conserved in midge Dscam1. Studies have shown that the docking site of the Dscam 1 exon 6 cluster is conserved in fly and crustacean water fleas, spanning $\sim 20$ million years of evolution (Fig. 2A; Graveley 2005; Brites et al. 2008; May et al. 2011). This may represent one of the most conserved ciselements known in invertebrates. However, when we subsequently scanned the intron downstream from exon 5 in midge Dscam 1 with the ultra-conserved sequence, unexpectedly we were unable to find any similarity to the fly docking site. This is of great interest, because comparative analysis revealed that the docking sites of the Dscam 1 exon 6 cluster were conserved in almost all major taxonomic groups of the investigated insects and crustaceans (Fig. 2A; Supplemental Table S1). Likewise, we failed to find selector sequences in the midge exon 6 cluster, while several selector sequences of the exon 6 cluster are conserved in dipteran and silkworm species. Therefore, we speculate that the docking site and selector sequence were specifically changed in midge species. Alternatively, independent duplications in midge species created a completely different set of docking-selector sequences as previously proposed (Ivanov and Pervouchine 2018).

Remarkably, sequence comparison revealed one highly conserved sequence in the intron downstream from the constitutive exon 5 between the two midge species ( $B$. antarctica and C. marinus) (Fig. 2A). Likewise, one relatively conserved sequence resided in the intron upstream of some exon 6s, such as exon 6.2 and 6.3 (Fig. 2C). These two types of midge conserved intronic elements did not exhibit any similarity to the docking site and selector sequences of the fly exon cluster 6 . However, the docking site is strongly complementary to the predicted selector sequences upstream of several exon 6 s, such as $C$. marinus 
A

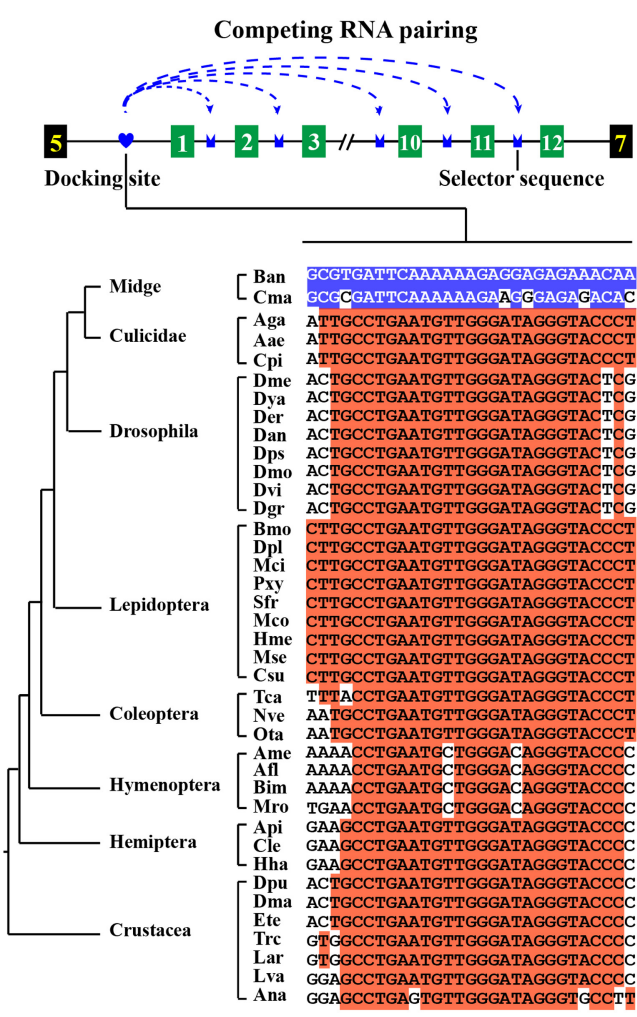

B

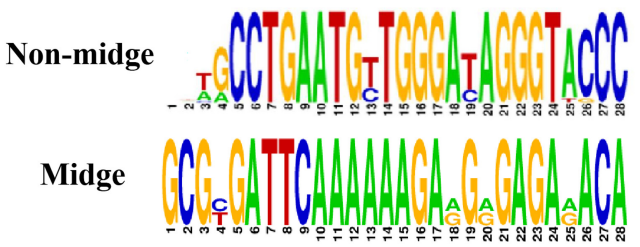

C

6.2

6.5

6.11

6.12

GUCAGGCGCGAUUCAA ${ }^{\mathrm{A}}$ AAAG ${ }^{\mathrm{A}}$ AGGGAGAGACACACAAGAAA AAGUU ${ }_{C}$ UUUC UC UCUCUCUUUGUGUGUUC

6.15

\section{B. antarctica}

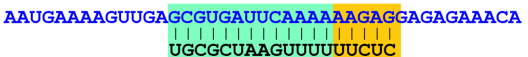

AAUGAAAAGUUGAGCGUGAUUCAAAAAAGAGGAGAGAAACA UUGCUUUUUAGUUCGCGCUAAG

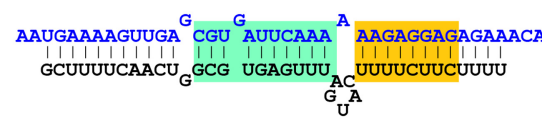

AAUGAAAAGUUGAGCGUGAUU CA AAAAGAGGAG AGAAACA CGUAUUAG II $G$ UI II IIII II II III

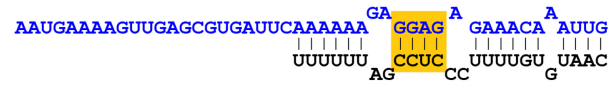

\section{C. marinus}

GUC AGGCGCGAUUCAAAAAAGAAGGGAGAGACACACAAGAAA UAG UUUGCGCUAAGUUUU

\section{GUCAGGCGCGAUUCAAAAAAGAAGGGAGAGACACACAAGAAA}

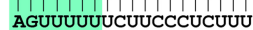

GUCAGGC GCGAUUCAAAAAAAGAA GGAGAGACACACAAGAAA UCG CGCUAAGUUUUUUU⿴囗十

GUCAGGCGCGAUUCAAAAAAGAAGGGA GAG CACACAAGAAAU CUUCCCU CA CUC GUGUGUUCUUUG ${ }_{A_{A} G}^{C A}$

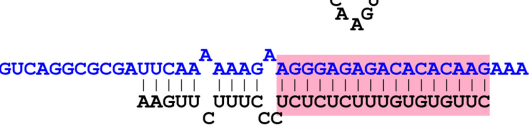

GUCAGGCGCGAUUCAAAAAAGAAGGGAGAGACA ACAA GAAA UUCUUUCUUCUCUGU UGUU ${ }_{\mathrm{AG}}$ CUUU

FIGURE 2. RNA secondary structures in midge exon 6 cluster. (A) The docking site of exon 6 cluster conserved in almost all insects and crustaceans is specific in midge. Schematic diagram of the exon 6 cluster in the midge B. antarctica was shown. Docking site (marked by hearts) was reverse complementary to a selector sequence (marked by crowns). The docking site of Dscam 1 exon 6 cluster is conserved in all major taxonomic groups of insects and crustaceans, except for the midge. (B) Comparison of docking site sequence consensus between the midge and other insect and crustacean species. (C) Base-pairings between the docking site and selector sequence are predicted in the exon 9 of Dscam1 of midge (B. antarctica and $C$ marinus). The relatively conserved stems of base-pairings among variable exons of different midges are highlighted in color.

exon 6.2, 6.3, 6.6, 6.14, and 6.15 (Fig. 2C; Supplemental Fig. S4). These RNA secondary structures partly share the core stem of base-pairings among variable exons of different midge species. Thus, we concluded that the docking site and selector sequences of midge Dscam 1 are species-specific at the sequence level, but evolutionarily conserved at the secondary-structural level. Moreover, RNA secondary structures will still need to be experimentally verified with disrupting and compensatory mutations. However, when we attempted to generate the Antarctic midge minigene construct for transfection experiments with Drosophila S2 cells, almost all exon 6 variants were not included. Therefore, we did not verify these proposed RNA secondary structures. However, their apparent evolutionary conservation combined with previous experimen- tal evidence in the exon cluster 6 of Drosophila Dscam 1 (May et al. 2011) suggests that these cis-elements mainly function through the formation of long-range basepairings.

\section{Competing RNA secondary structures are apparent in the midge exon 9 cluster}

To determine whether the docking site-selector base-pairings are conserved in the exon 4 and 9 clusters of midge Dscam1, we scanned the corresponding sequence of midge Dscam1 using the docking site and selector sequences identified in other species. However, we were unable to find any similar sequences in the midge exon 4 and 9 cluster. Instead, we found two types of midge-conserved 
docking site and selector sequences in the midge exon 9 cluster. Strikingly, the consensus selector sequence was complementary to the docking site sequence (Fig. 3A,B). Thus, the docking site and selector sequences of the midge exon 9 cluster are species-specific at the sequence level, but evolutionarily conserved at the secondary-structural level. However, similar docking site-selector basepairings were not readily apparent in the exon 4 cluster of midge Dscam1. Due to technical challenges associated with the large size and complexity of the midge exon 9 cluster (>11 kb), we did not verify these proposed RNA secondary structures. However, their apparent evolutionary conservation combined with previous experimental evidence in the exon cluster 9 of silkworm Dscam 1 (Yue et al. 2016b) suggest that the docking site-selector base-pairings are crucial players in regulating exon 9 alternative splicing.

Interestingly, we found that all 21 predicted selector sequences were strongly complementary to the docking site in the midge species B. antarctica (Fig. 3A). Similar docking site-selector base-pairings were shown in the midge species C. marinus (Supplemental Fig. S5). Thus, the architecture of the docking site-selector base-pairing in the midge exon 9 cluster is analogous to that of the $D$. melanogaster exon 6 cluster. Notably, the D. melanogaster exon 6 cluster exhibits a broad spectrum of expression of the variable exon 6s (Neves et al. 2004; Watson et al. 2005; Sun et al. 2013). Likewise, we also observed a broad spectrum of expression of the variable exon $9 \mathrm{~s}$ in the midge based on RNA sequencing data (Fig.4B), compatible with the observation that base-pairings reside downstream from almost every variable exon 9 of the midge Dscam1. In contrast, D. melanogaster exon 9 exhibited highly restricted expression (Neves et al. 2004; Watson et al. 2005; Sun et al. 2013), compatible with the observation that these apparent base-pairings reside downstream from a few predominantly expressed variable exon 9s (Hong et al. 2019). This suggests a potential mechanistic link between expression pattern and the architecture of the base-pairing between the docking site and selector sequence.

Our results conflict with a recent study, which argued that a small complement of regulatory factors act as the main determinants of exon inclusion in the Dscam 1 exon 9 cluster (Ustaoglu et al. 2019). The authors attributed this to the absence of long-range base-pairings in the
A

\section{B. antarctica selector sequences}

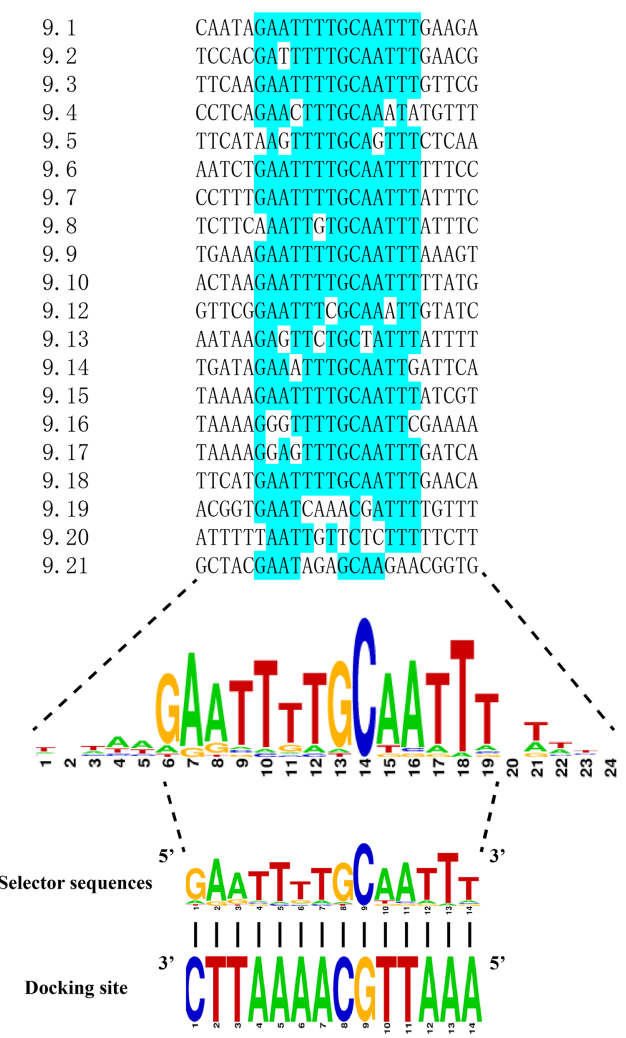

B

\section{C. marinus selector sequences}

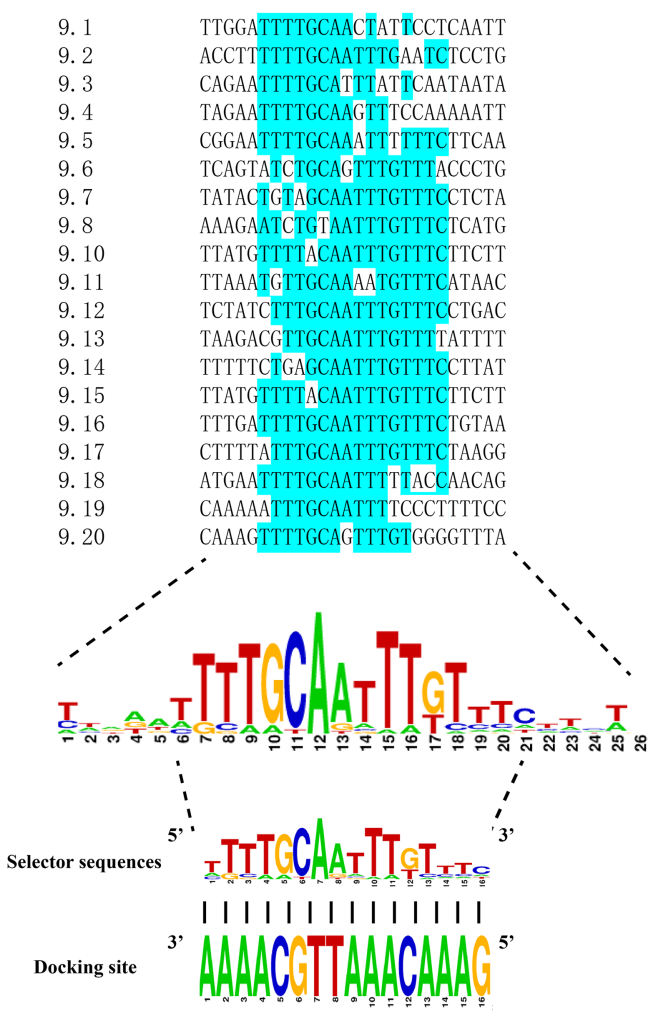

FIGURE 3. The consensus selector sequence is complementary to the docking site of exon 9 cluster of midge Dscam1. All selector sequences were aligned together in two midges, $B$. antarctica $(A)$ and $C$. marinus $(B)$, respectively. The most frequent nucleotides in the central region of the alignment are highlighted in blue. The consensus nucleotides of the selector sequences were complementary to the docking site. 

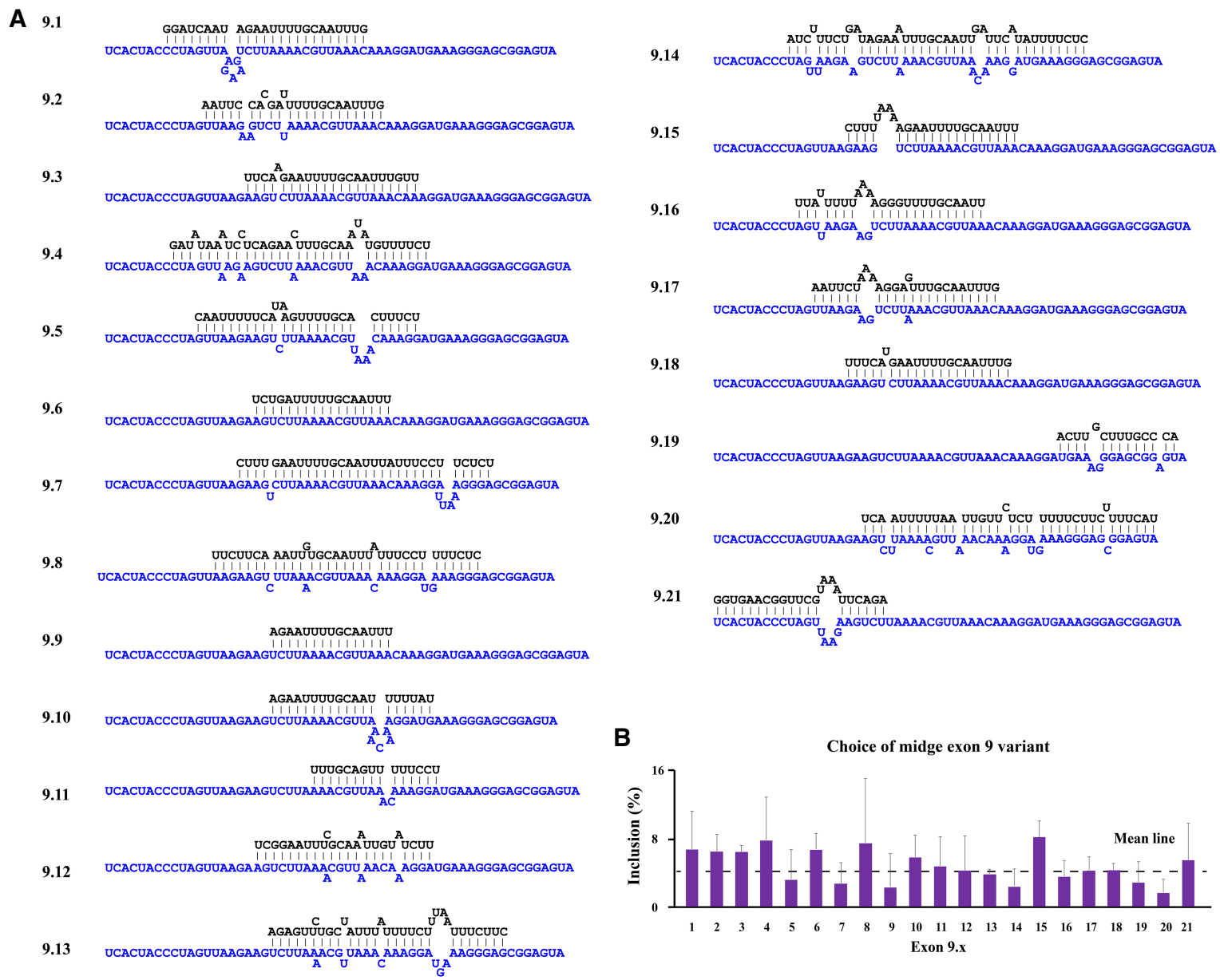

FIGURE 4. Docking site-selector base-pairings and the choice of exon 9 variant in midge. (A) Predicted base-pairings between the docking site and selector sequence are shown for $B$. antarctica exon 9 cluster. The selector sequence resided downstream from almost every variable exon 9 of Dscam 1 of Dipteran midge potentially paired strongly with the docking site. (B) The choice of B. antarctica exon 9 variants. The expression level for each isoform was calculated based on RNA-seq data from midge whole body. Data are expressed as a percentage of the mean \pm SD from three independent experiments. The selector sequence downstream from almost each variable exon 9 of midge potentially paired strongly with the docking site, which is consistent with the broad spectrum of isoform expression.

variable exon 9 cluster. Apparent evolutionary signatures in the present study clearly support competing RNA secondary structures in the exon cluster 9 of the midge Dscam1 gene. Furthermore, we hypothesize that if the evolutionary conserved sequences act to bind regulatory proteins, they are likely to contain similar or common motifs. However, the docking sites and selector sequences in the Dscam 1 exon 9 cluster in Drosophila, Lepidoptera, and Hymenoptera are completely different (Yang et al. 2011; Yue et al. 2016b). The present study also shows that the docking sites and selector sequences of the exon 6 and 9 clusters in Drosophila and midge Dscam 1 are completely different. Importantly, compensatory structure-restoring mutations reveal that these elements function via the formation of non-sequence-specific RNA pairing (May et al. 2011; Yang et al. 2011; Yue et al. 2016b). Therefore, we speculate that such types of cis-elements mainly function via the formation of non-sequence-specific RNA pairing.

\section{Conclusions}

This study provides evident and unique evolutionary signatures that support mutually exclusive splicing mediated by competing RNA pairing in the given two midge species. Dscam 1 in these midge species encodes only 6000 different isoforms, representing the smallest splice repertoire known among insects to date. Strikingly, the docking site of the exon 6 cluster is conserved in almost all insects and crustaceans, but exhibits marked differences in the midge species. However, their docking site and selector sequences are evolutionarily conserved at the secondarystructural level. Moreover, the docking site-selector base-pairing was located downstream from almost each variable exon 9 of the midge Dscam1, which is in accordance with the broad spectrum of their isoform expression. Overall, these findings suggest that these types of cis-elements mainly function through the formation of long- 
range base-pairings. This study provides vital insights into the evolution and mechanism of alternative splicing.

\section{MATERIALS AND METHODS}

\section{Identification and annotation of Dscam1 orthologs}

The nucleotide sequences of Dscam 1 genes were obtained from previous studies (Supplemental Table S1; Schmucker et al. 2000; Graveley 2005; Brites et al. 2008; May et al. 2011). The Dscam1 genes from the two midges ( $B$. antarctica and $C$. marinus) were obtained by TBLASTN searches of the NCBI WGS database (http://blast.ncbi.nlm.nih.gov/Blast.cgi). The exons and introns of Dscam 1 genes were predicted by manual inspection of the nucleotide sequences. However, sometimes we obtained RNA-seq data and some data of cDNA from NCBI database, which helped us to define the Dscam 1 gene structures accurately.

\section{Sequence alignment and phylogenetic analysis}

Alignments of amino acid encoding variable exons among species were carried out using ClustalW (https://www.genome.jp/ tools-bin/clustalw). Nucleotide sequences were aligned using MUSCLE program according to the system default parameters. The phylogenetic tree was constructed using MEGA X (https:// www.megasoftware.net/). The tree was reconstructed using a Nearest-Neighbor-Interchange (NNI) method based on "maximum composite likelihood" model. The phylogeny was rooted on midpoint. The RNA base-pairing interactions between the docking site and the selector sequences were predicted using the Mfold program (Zuker 2003). The consensus sequences of the selector sequences were derived using WebLogo (http:// weblogo.berkeley.edu/logo.cgi) (Crooks et al. 2004).

\section{Analysis of RNA-seq data}

We calculated the relative expression levels of variable exon 9s of the Dipteran midge ( $B$. antarctica) according to the method as previously described (Yue et al. 2016a). The expression level for each isoform was calculated using three RNA-seq data (SRX185013, SRX185021, SRX185620). Data are expressed as a percentage of the mean $\pm S D$ from three independent experiments.

\section{Minigene construction, mutagenesis, and transfection}

The exon 6 minigene was constructed to encompass the constitutive exon 5 , the constitutive exon 7 , and the entire intervening sequence. Genomic DNAs isolated from the midge B. antarctica (gift from Nicholas M. Teets, the University of Kentucky) were used as templates to obtain the corresponding DNA segment with PCR. The fragment DNA was confirmed by DNA sequencing and then cloned into the pMT/V5-His B vector (Invitrogen) with the metallothionein promoter. Transfection experiments were performed as previously described (Yang et al. 2011).

\section{SUPPLEMENTAL MATERIAL}

Supplemental material is available for this article.

\section{ACKNOWLEDGMENTS}

This work was supported by research grants from the National Natural Science Foundation of China (91740104, 91940303, 31630089).

Received March 5, 2020; accepted May 27, 2020

\section{REFERENCES}

Anastassiou D, Liu H, Varadan V. 2006. Variable window binding for mutually exclusive alternative splicing. Genome Biol 7: R2. doi:10.1186/gb-2006-7-1-r2

Brites D, McTaggart S, Morris K, Anderson J, Thomas K, Colson I, Fabbro T, Little TJ, Ebert D, Du Pasquier L. 2008. The Dscam homologue of the crustacean Daphnia is diversified by alternative splicing like in insects. Mol Biol Evol 25: 1429-1439. doi:10 .1093/molbev/msn087

Crooks GE, Hon G, Chandonia JM, Brenner SE. 2004. WebLogo: a sequence logo generator. Genome Res 14: 1188-1190. doi:10 $.1101 /$ gr.849004

Graveley BR. 2005. Mutually exclusive splicing of the insect Dscam pre-mRNA directed by competing intronic RNA secondary structures. Cell 123: 65-73. doi:10.1016/j.cell.2005.07.028

Hatje K, Rahman RU, Vidal RO, Simm D, Hammesfahr B, Bansal V, Rajput A, Mickael ME, Sun T, Bonn S, et al. 2017. The landscape of human mutually exclusive splicing. Mol Syst Biol 13: 959. doi:10.15252/msb.20177728

Haussmann IU, Ustaoglu P, Brauer U, Hemani Y, Dix TC, Soller M. 2019. Plasmid-based gap-repair recombineered transgenes reveal a central role for introns in mutually exclusive alternative splicing in Down Syndrome Cell Adhesion Molecule exon 4. Nucleic Acids Res 47: 1389-1403. doi:10.1093/nar/gky1254

He H, Kise Y, Izadifar A, Urwyler O, Ayaz D, Parthasarthy A, Yan B, Erfurth ML, Dascenco D, Schmucker D. 2014. Cell-intrinsic requirement of Dscam1 isoform diversity for axon collateral formation. Science 344: 1182-1186. doi:10.1126/science.1251852

Hong W, Dong H, Zhang J, Zhou F, Wu Y, Shi Y, Chen S, Xu B, You W, Shi $F$, et al. 2019. Intron-targeted mutagenesis reveals roles for Dscam1 RNA pairing-mediated splicing bias in neuronal wiring. bioRxiv doi:10.1101/622217

Ivanov T, Pervouchine D. 2018. An evolutionary mechanism for the generation of competing RNA structures associated with mutually exclusive exons. Genes (Basel) 9: 356. doi:10.3390/ genes9070356

Kelley JL, Peyton JT, Fiston-Lavier AS, Teets NM, Yee MC, Johnston JS, Bustamante CD, Lee RE, Denlinger DL. 2014. Compact genome of the Antarctic midge is likely an adaptation to an extreme environment. Nat Commun 5: 4611. doi:10.1038/ ncomms 5611

Lee Y, Rio DC. 2015. Mechanisms and regulation of alternative premRNA splicing. Annu Rev Biochem 84: 291. doi:10.1146/ annurev-biochem-060614-034316

Lee C, Kim N, Roy M, Graveley BR. 2010. Massive expansions of Dscam splicing diversity via staggered homologous recombination during arthropod evolution. RNA 16: 91-105. doi:10.1261/ rna.1812710

Matera AG, Wang Z. 2014. A day in the life of the spliceosome. Nat Rev Mol Cell Biol 15: 108-121. doi:10.1038/nrm3742 
May GE, Olson S, McManus CJ, Graveley BR. 2011. Competing RNA secondary structures are required for mutually exclusive splicing of the Dscam exon 6 cluster. RNA 17: 222-229. doi:10.1261/rna .2521311

Neves G, Zucker J, Daly M, Chess A. 2004. Stochastic yet biased expression of multiple Dscam splice variants by individual cells. Nat Genet 36: 240-246. doi:10.1038/ng1299

Nilsen TW, Graveley BR. 2010. Expansion of the eukaryotic proteome by alternative splicing. Nature 463: 457-463. doi:10.1038/ nature08909

Olson S, Blanchette M, Park J, Savva Y, Yeo GW, Yeakley JM, Rio DC, Graveley BR. 2007. A regulator of Dscam mutually exclusive splicing fidelity. Nat Struct Mol Biol 14: 1134-1140. doi:10.1038/ nsmb1339

Pan H, Shi Y, Chen S, Yang Y, Yue Y, Zhan L, Dai L, Dong H, Hong W, Shi $F$, et al. 2018. Competing RNA pairings in complex alternative splicing of a 3'variable region. RNA 24: 1466-1480. doi:10.1261/ rna.066225.118

Schmucker D, Clemens JC, Shu H, Worby CA, Xiao J, Muda M, Dixon JE, Zipursky SL. 2000. Drosophila Dscam is an axon guidance receptor exhibiting extraordinary molecular diversity. Cell 101: 671-684. doi:10.1016/S0092-8674(00)80878-8

Scotti MM, Swanson MS. 2016. RNA mis-splicing in disease. Nat Rev Genet 17: 19-32. doi:10.1038/nrg.2015.3

Sun W, You X, Gogol-Doring A, He H, Kise Y, Sohn M, Chen T, Klebes A, Schmucker D, Chen W. 2013. Ultra-deep profiling of alternatively spliced Drosophila Dscam isoforms by circularizationassisted multi-segment sequencing. EMBO J 32: 2029-2038. doi:10.1038/emboj.2013.144

Suyama M. 2013. Mechanistic insights into mutually exclusive splicing in dynamin 1. Bioinformatics 29: 2084-2087. doi:10.1093/bioinfor matics/btt368

Ustaoglu P, Haussmann IU, Liao H, Torres-Mendez A, Arnold R, Irimia M, Soller M. 2019. Srrm234, but not canonical SR and hnRNP proteins, drive inclusion of Dscam exon 9 variable exons. RNA 25: 1353-1365. doi:10.1261/rna.071316.119
Wang $X$, Li G, Yang Y, Wang W, Zhang W, Pan H, Zhang P, Yue Y, Lin H, Liu B, et al. 2012. An RNA architectural locus control region involved in Dscam mutually exclusive splicing. Nat Commun 3: 1255. doi:10.1038/ncomms2269

Wang Y, Bao Y, Zhang S, Wang Z. 2020. Splicing dysregulation in cancer: from mechanistic understanding to a new class of therapeutic targets. Sci China Life Sci 63: 469-484. doi:10.1007/s11427-0191605-0

Watson FL, Puttmann-Holgado R, Thomas F, Lamar DL, Hughes M, Kondo M, Rebel VI, Schmucker D. 2005. Extensive diversity of Ig-superfamily proteins in the immune system of insects. Science 309: 1874-1878. doi:10.1126/science.1116887

Xu B, Shi Y, Wu Y, Meng Y, Jin Y. 2019. Role of RNA secondary structures in regulating Dscam alternative splicing. Biochim Biophys Acta Gene Regul Mech 1862: 194381. doi:10.1016/j.bbagrm .2019 .04 .008

Yang Y, Zhan L, Zhang W, Sun F, Wang W, Tian N, Bi J, Wang H, Shi D, Jiang $Y$, et al. 2011. RNA secondary structure in mutually exclusive splicing. Nat Struct Mol Biol 18: 159-168. doi:10.1038/nsmb.1959

Yue Y, Meng Y, Ma H, Hou S, Cao G, Hong W, Shi Y, Guo P, Liu B, Shi $F$, et al. 2016a. A large family of Dscam genes with tandemly arrayed $5^{\prime}$ cassettes in Chelicerata. Nat Commun 7: 11252. doi:10.1038/ncomms11252

Yue Y, Yang Y, Dai L, Cao G, Chen R, Hong W, Liu B, Shi Y, Meng Y, Shi $F$, et al. 2016b. Long-range RNA pairings contribute to mutually exclusive splicing. RNA 22: 96-110. doi:10.1261/rna.053314 .115

Yue Y, Hou S, Wang X, Zhan L, Cao G, Li G, Shi Y, Zhang P, Hong W, $\mathrm{Lin} H$, et al. 2017. Role and convergent evolution of competing RNA secondary structures in mutually exclusive splicing. RNA Biol 14: 1399-1410. doi:10.1080/15476286.2017.1294308

Zipursky SL, Sanes JR. 2010. Chemoaffinity revisited: Dscams, protocadherins, and neural circuit assembly. Cell 143: 343-353. doi:10 .1016/j.cell.2010.10.009

Zuker M. 2003. Mfold web server for nucleic acid folding and hybridization prediction. Nucleic Acids Res 31: 3406-3415. doi:10.1093/ nar/gkg595 

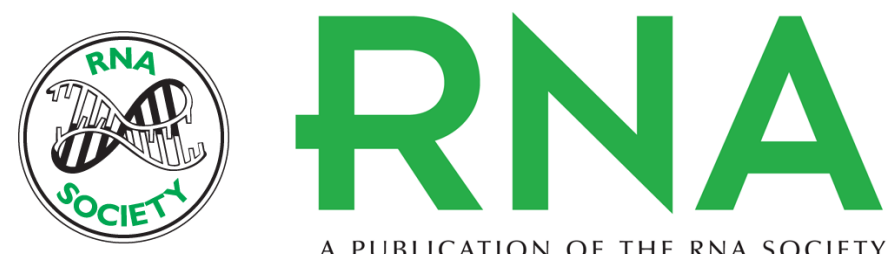

A PUBLICATION OF THE RNA SOCIETY

\section{RNA secondary structures in Dscam1 mutually exclusive splicing: unique evolutionary signature from the midge}

Weiling Hong, Yang Shi, Bingbing Xu, et al.

RNA 2020 26: 1086-1093 originally published online May 29, 2020

Access the most recent version at doi:10.1261/rna.075259.120

\section{Supplemental http://rnajournal.cshlp.org/content/suppl/2020/05/29/rna.075259.120.DC1 \\ Material}

References This article cites 33 articles, 11 of which can be accessed free at: http://rnajournal.cshlp.org/content/26/9/1086.full.html\#ref-list-1

Creative This article is distributed exclusively by the RNA Society for the first 12 months after the Commons full-issue publication date (see http://rnajournal.cshlp.org/site/misc/terms.xhtml). After 12

License months, it is available under a Creative Commons License (Attribution-NonCommercial 4.0 International), as described at http://creativecommons.org/licenses/by-nc/4.0/.

Email Alerting Receive free email alerts when new articles cite this article - sign up in the box at the Service top right corner of the article or click here.

\section{|||||||| Providing Precise Solutions for your research.}

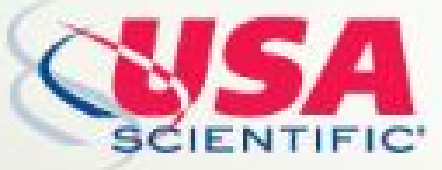

To subscribe to $R N A$ go to:

http://rnajournal.cshlp.org/subscriptions 